\title{
ASSOCIATION OF CHROMOSOMAL ABERRATIONS AND SEMEN QUALITY IN EGYPTIAN BUFFALO BULLS
}

\author{
H. E. Rushdi ${ }^{1 *}$, M. F. Saad ${ }^{2}$, H. A. El-naggar ${ }^{2}$ and A. M. Saeed ${ }^{2}$ \\ 1- Department of Animal Production, Faculty of Agriculture, Cairo University, Giza, 12613, Egypt, 2- \\ Department of Animal Biotechnology, Animal Production Research Institute, Dokki, Giza, Egypt. \\ *Corresponding Author's E-mail: hosamrushdi@yahoo.com hosamrushdi@agr.cu.edu.eg, Telephone: + 202 \\ 3378 6729, Fax: + 2023569 4049, Cellular phone: + 201223409079
}

\begin{abstract}
SUMMARY
Milk production and processing in Egypt is a basically function of buffalo and cattle. Various reasons justify sterility and low reproductive performance in livestock, among them chromosomal aberration which is highly considered. The present study was undertaken to investigate the association between semen characteristics and cytogenetic structure as an early indicator of fertility in Egyptian buffalo bulls. Data and samples were collected during September and October, 2015 on 116 ejaculates of 29 randomly selected bulls (age from 22 to 26 months and body weight from 250 to $300 \mathrm{~kg}$ ). Animals were classified into two groups based on semen quality: group 1 (G1) composed of 10 animals with low semen quality characteristics and group 2 (G2) that included 19 bulls with normal semen quality characteristics. Polyploidy accounted for 9.6\% of total numerical aberrations in G1 compared to $1.2 \%$ in G2, while aneuploidy represented $5 \%$ and $0.4 \%$ in G1 and G2, respectively. $G 1$ and $G 2$ differed significantly $(P<0.05)$ in the percent of polyploidy and aneuploidy. Structural chromosomal aberrations identified were deletion, break, gap, fragmentation, ring, centromeric attenuation and centric fusion, which varied significantly $(P<0.05)$ between $G 1$ and $G 2$. Such aberrations in metaphase chromosomes may have a direct relevance on fertility as they were consistent. All estimates of genetic correlation were significant $(P<0.05)$, except that were related to centromeric attenuation. Chromosomal aberrations correlated negatively with motility, live sperm, hypo-osmotic swollen (\%) and testosterone level; whereas their correlation coefficients with sperm abnormality were positive. Genetic correlations ranged from 0.391 between motility and polyploidy to 0.341 between sperm abnormality and polyploidy. The results obtained suggest screening buffalo bulls for chromosomal aberrations prior to their use in natural or artificial insemination.
\end{abstract}

Keywords: Buffalo, breeding bulls, correlation; chromosomal abnormalities, semen quality

\section{INTRODUCTION}

The global population of water buffalo has increased $121 \%$ during the last few decades, from 88 million in 1961 to 195 million in 2014 (FAOSTAT, 2016). Buffalo plays a vital role in agriculture and food security, where they are extensively used for milk, meat, fuel and fertilizer production, as well as for draught power, especially for the poorest rural families in developing countries. With this large population of buffalo which is very well adapted to the tropical and subtropical environments and has a competitive potential to meet the protein requirement of our growing human population, it appears quite appropriate to call it "black gold".

Buffalo contributes $70 \%$ of the total milk production in Egypt. In spite of Egypt's position as the fourth highest producer of buffalo milk in the world preceded only by India, Pakistan and China, productivity per animal is poor and not yet suitable for intensive commercial dairy farming systems, as compared to dairy cattle (FAOSTAT, 2016; http://faostat.fao.org). This is mainly due to low genetic potential for milk production, absence of confident farm records, using animals in agricultural works, poor level of nutrition and health care as well as limited reproductive performance (Galal, 2007). Fortunately, there is still a large room to genetically improve buffalo productivity through information produced from genomic studies and scientific research.

Traditionally, genetic improvement of livestock has widely laid on selection of animal candidates to be parent to next generations. Selection based on chromosomal morphology composes the first step to implement schemes and strategies for the genetic improvement of quantitative traits in Egyptian buffaloes. Chromosomal aberrations, detected by cytogenetic studies, are usually associated with reproductive disorder in livestock (Jamir et al., 2015). Therefore, animal cytogenetics provides a powerful basis for decision-making process towards culling pedigree-selected animals for breeding with chromosomal aberrations at an earlier age, which subsequently saves time, reduces cost of rearing, feeding and health care of animals, and also increases herd's profitability (Yimer and Rosnina, 2014). On the basis of these consequences, screening breeding bulls for chromosomal aberrations is recommended.

Several studies on clinical cytogenetics have been carried out in cattle suggesting chromosomal abnormalities as a reason of sterility and low reproductive performance due to unbalanced gametes during meiosis division (Patel et al., 2012; Jamir et al., 2015 and Zhang et al., 2015). Also, different chromosomal aberrations affecting fertility have been reported in buffaloes (Vijh et al., 1994; Ahmad et al., 2004; Chauhan et al., 2009; Raudsepp and 
Chowdhary, 2016 and Mughal et al., 2017). Fertility is a multifactorial phenomenon which is influenced by several factors that often overlap including nutrition, disease, hormonal interactions, reproductive functions, sperm quality, physical soundness, environment, management, genetic factors and abnormal karyotypes that lead to lower reproductive performance of farm animals through decreasing or forbidding capability to produce functional gametes as well as death of early- and latematurity embryos (Yimer and Rosnina, 2014). The authors explained that those types of aberrations were gap formation (the lost area is shorter than diameter of chromatid), chromosome break (the lost area is longer than the diameter of chromatid or equal to it), chromosome deletion (one chromatid shorter than its sister), centromeric attenuations (the arm of one chromatid is separated from its sister), centric fusion (one chromosome is fused with another chromosome by centromers) and ring chromosome (the ends of the two chromosome's arms are joined together). However, information associating chromosomal morphology and structure with fertility of Egyptian buffalo bulls is scarce.

Therefore, the objectives of the present study were to identify chromosomal abnormalities in breeding bulls of Egyptian buffalo and to evaluate their possible association with semen quality characteristics.

\section{MATERIALS AND METHODS}

\section{Animals and semen collection:}

Resource population of the present study was based on 29 sexually mature bulls of Egyptian water buffalo. Age of animals was between 22 and 26 months old and their live body weight ranged from 250 to $300 \mathrm{~kg}$. All bulls involved were free of any clinical diseases with a healthy appearance. Semen collection was conducted by means of an artificial vagina set up at optimal conditions to induce a good ejaculatory thrust. At the time of semen collection, another buffalo bull was used as a teaser for sexual preparation. Ejaculates were obtained from each bull one time per week at early morning (7:00am) for 4 consecutive weeks during September and October, 2015 , resulted in a total number of 116 samples. The collected ejaculates were taken immediately to the laboratory in water bath at $37^{\circ} \mathrm{C}$ for semen evaluation processes.

\section{Progressive sperm motility (\%):}

The percentage of motile spermatozoa was assessed using light microscope supplied with a hot stage adjusted to $37^{\circ} \mathrm{C}$. Aliquots of evaluated semen were placed on the slide and covered by a warmed cover slip and were immediately examined under a high power magnification (400x) according to Amann and Hammerstedt (1980).

\section{Live sperm (\%):}

A smear from semen was made on a glass slide and stained by $1.67 \%$ eosin (E 8761) and $10 \%$ nigrosin ( $\mathrm{N} 4763$ ), a mixture stain proposed by
Hackett and Macpherson (1965). Except mentioned differently, all experimental reagents used were purchased from Sigma-Aldrich (S.A., Egypt). About $10 \mathrm{~g}$ Nigrosin and $1.67 \mathrm{~g}$ Eosin were dissolved in distilled water up to $100 \mathrm{ml}$ for the preparation of Eosin-Nigrosin stain at $37^{\circ} \mathrm{C}$. One drop of the prepared stain was added to one drop of the fresh semen and was mixed on glass slide at $37^{\circ} \mathrm{C}$. Then, a thin smear was made by drawing of a second slide across the stained semen. The slide was allowed to dry on the hot stage and then examined under a high power magnification $(400 \mathrm{x})$. At the examination time, the live spermatozoa (unstained ones) and dead spermatozoa (stained ones) were counted in field of a total of 200 spermatozoa.

\section{Sperm abnormalities (\%):}

During the examination of live/dead sperm percentage at a high power magnification (x400), the morphological screening of spermatozoa was carried out per 200 spermatozoa according to the classification adopted by Blom (1983).

\section{Hypo-osmotic swelling test (HOST):}

The response of spermatozoa to HOST was assessed using solution prepared with $0.21 \%$ lactose (L7401) and $0.48 \%$ sodium citrate $(\mathrm{N} 3269)$ in a distilled water to give osmolarity of $50 \mathrm{ml}$ osmol using a freezing-point depression Osmometer (Osmett A, Model 5002, Fisher Scientific, Pittsbury, PA, USA). Immediately after thawing, semen aliquots of $0.1 \mathrm{ml}$ were added to $0.9 \mathrm{ml}$ a mixture of sodium-citrate-lactose solution. The mixture was incubated at $37^{\circ} \mathrm{C}$ for $30 \mathrm{~min}$. A drop from the mixture was placed on a warmed glass slide at $37^{\circ} \mathrm{C}$ and cover slipped. At least 200 spermatozoa were examined for calculating the percentage of curled spermatozoa, according to the method suggested by Azam et al. (1998).

\section{Hormone measurement:}

Blood samples were collected through jugular vein of each animal into heparinized vacutainer tube. After an hour at room temperature, samples were centrifuged at $2000 \mathrm{rpm}$ for $20 \mathrm{~min}$, and then plasma was collected and immediately kept frozen at $-20{ }^{\circ} \mathrm{C}$ until the hormone assay. Concentration of the testosterone hormone was determined by radioimmunoassay according to the method described by Smith and Hafs (1973).

\section{Chromosomal preparation and analysis:}

Under hygienic condition, a blood sample of three $\mathrm{ml}$ was collected from the jugular vein of each animal into a sterilized sodium-heparinizedvacutainer tube with special care in handling. Chromosomal preparations were performed using standard whole blood culture in tissue culture 199 medium (TCM 199) (M4531) supplemented with antibiotics (Streptomycin and pencillin) (S2522), $20 \%$ fetal calf serum (F2442), $0.1 \%$ phytoheamagglutanin and 0.1\% L-Glutamin (G7513) were added to stimulate mitotic activity of the lymphocyte cells and to facilitate the identification of 
chromosomes. The blood culture was incubated at $37^{\circ} \mathrm{C}$ for 72 hours. To increase the relative frequency of prometaphase chromosomes, Colchicine (C180) 1 $\mu \mathrm{g} / \mathrm{ml}$ was added to the culture for two hours prior to the harvesting. The cells were collected by centrifugation at $1500 \mathrm{~g}$ for seven min, followed by hypotonic treatment with $0.56 \%$ potassium chloride (P1302) for $20 \mathrm{~min}$ at $37^{\circ} \mathrm{C}$ and then fixed in $3: 1$ ratio of methanol (M0789) and glacial acetic acid. Finally, cell suspension was dropped on slides and air dried. Slides were conventionally stained in $10 \%$ Giemsa stain (G9641) for screening under the Nikon compound microscope attached with photographic system. About 50 metaphase spreads were screened to detect the chromosomal aberrations and prepare the karyotype (Halnan, 1977 and Iannuzzi and Di Berardino, 2008).

\section{Statistical analysis:}

Based on semen quality, breeding bulls were classified into two groups: group 1 (G1) composed of 10 animals with low semen quality characteristics and group 2 (G2) that included 19 bulls with normal semen quality characteristics. $\mathrm{T}$ test model was performed with SPSS 16.0 software package (SPSS Inc., Chicago, Illinois, USA, 2006). Results are expressed as means \pm standard error of the mean. The level of significance adopted was set at $\mathrm{P}<0.05$.

Pearson's correlation coefficients were applied to examine the relationship between semen characteristics and chromosomal aberrations. Estimates of genetic correlation were considered statistically significant at $\mathrm{P}<0.05$.

\section{RESULTS AND DISCUSSION}

\section{Semen characteristics:}

Physical characteristics of buffalo semen are illustrated in Table (1). In general, means of the semen parameters obtained in the present study are comparable to those reported in the Egyptian river buffalo bulls by Hafez (2012), Khattab et al. (2015) and Mahmoud et al. (2015) and also in swamp buffalo as mentioned by Koonjaenak et al. (2007) and Bhakat et al. (2015).

Table 1. Physical semen characteristics, hypo-osmotic swelling test and testosterone hormone level in Egyptian buffalo bull semen (mean \pm S.E.)

\begin{tabular}{|c|c|c|c|c|c|c|}
\hline \multirow{2}{*}{ Group } & \multirow{2}{*}{$\begin{array}{c}\text { No. of } \\
\text { animals }\end{array}$} & \multicolumn{3}{|c|}{ Semen characteristics $(\%)$} & \multirow{2}{*}{$\begin{array}{c}\text { Hypo-osmotic } \\
\text { swollen (\%) }\end{array}$} & \multirow{2}{*}{$\begin{array}{l}\text { Testosterone } \\
\text { level (ng/ml) }\end{array}$} \\
\hline & & Motility & Live & Abnormality & & \\
\hline G1 & 10 & $53.37 \pm 0.70^{\mathrm{a}}$ & $57.57 \pm 0.52^{\mathrm{a}}$ & $20.35 \pm 0.27^{\mathrm{a}}$ & $50.42 \pm 0.69^{\mathrm{a}}$ & $1.55 \pm 0.06^{\mathrm{a}}$ \\
\hline $\mathrm{G} 2$ & 19 & $72.96 \pm 0.49^{b}$ & $77.43 \pm 0.36^{\mathrm{b}}$ & $12.42 \pm 0.31^{\mathrm{b}}$ & $71.29 \pm 0.42^{b}$ & $6.00 \pm 0.13^{b}$ \\
\hline $\begin{array}{l}\text { Overall } \\
\text { mean }\end{array}$ & 29 & $66.20 \pm 0.95$ & $70.58 \pm 0.93$ & $15.15 \pm 0.42$ & $64.09 \pm 0.99$ & $4.46 \pm 0.22$ \\
\hline
\end{tabular}

G1, low semen quality group; G2, normal semen quality group

Values with different superscripts within the same column differ significantly $(\mathrm{P}<0.05)$

Group 2 (G2) showed significantly $(\mathrm{P}<0.05)$ higher estimates in all studied semen traits than group 1 (G1), except abnormalities (\%). Percentages of motility and live sperm increased $(\mathrm{P}<0.05)$ by 36.71 and $34.50 \%$ in $\mathrm{G} 2$ compared with $\mathrm{G} 1$, respectively. In the same trend, hypo-osmotic swelling test (\%) and testosterone concentration $(\mathrm{ng} / \mathrm{ml})$ were also incremented by 41.39 and $287.1 \%$ in $\mathrm{G} 2$ in comparison to $\mathrm{G} 1$, respectively. On the other hand, abnormalities (\%) were decreased significantly $(\mathrm{P}<0.05)$ by 38.97 in $\mathrm{G} 2$ than $\mathrm{G} 1$.

The percent of motility obtained in the current study is very similar to the one found by Koonjaenak et al. (2007), who mentioned that the initial motility tended to be higher during winter rather than summer or rainy season. On the contrary, the overall mean of abnormalities (\%) detected in the present study was higher than the corresponding estimate reported by Bhakat et al. (2015) in Murrah buffalo (15.15 vs. $9.47 \%$, respectively). This finding may be caused by the collection of semen ejaculates during apparently hot months (September and October) along 4 consecutive weeks per bull, which resulted in increased abnormalities (\%). In summer season, extreme heat stress result in physical exhaustion, which might reduce the eagerness of bulls for copulation causing subsequently an increase in the reaction time and the total time for successful ejaculation, thus having a final effect on quantity and quality of sperms produced (Mandal et al., 2000).

Viability of sperms is related to the proportions of spermatozoa with an intact and osmotically functional plasma membrane (plasma membrane integrity, PMI) as mentioned by Barkawi et al. (2006) and Mughal et al. (2017). In the present study, PMI was measured by a hypo-osmotic swelling test (HOST). The percent of HOST obtained in the present study $(64.09 \%)$ is the same as that reported in Murrah buffalo by Koonjaenak et al. (2007), but it is higher than the value obtained for Nili-Ravi buffalo by Rasul et al. (2001). This difference could be attributed to the difference in animal species, environment and, most likely, to the method of measurement applied. The average PMI recorded using the HOST was significantly $(\mathrm{P}<0.05)$ higher in G2 compared to G1 (71.29 vs $50.42 \%$, respectively). Koonjaenak et al. (2007) reported seasonal variation in semen characteristics of swamp buffalo, where HOST estimates and the relative proportion of morphologically normal spermatozoa were significantly higher in summer compared to winter and rainy seasons. Although the proportions of PMI were influenced by season, they were always under what is considered refused for AI sires. In general, 
HOST is an effective and inexpensive technique to determine PMI of ejaculated spermatozoa. Variation in semen characteristics from month to another was also reported by Hafez (2012), Bhakat et al. (2015) and Saraswat and Purohit (2016). The evaluation of PMI holds promise as an early predictor of fertility as it has been stated that semen with negative HOST values had a poor fertilizing capacity (Capra et al., 2017). In addition, Jamir et al. (2015) reported significant differences between high and low motility groups in HOST scores; however, the HOST results did not differ significantly between cattle and buffalo. The results obtained in the present study suggest that the HOST can be used to judge the reproductive performance of breeding bulls and to differentiate the acceptable and unacceptable semen ejaculates. Recently, Mughal et al. (2017) concluded that osmotic pressure of the solution used in extender preparation influenced considerably post-thaw quality of cryopreserved buffalo bull semen.

In order to determine properly semen quality after sperm motility assessment, evaluation of sperm morphology is usually performed to identify sperm abnormalities which can reflect testicular, epididymal and accessory gland affections, and can also indicate any mishandling of the ejaculates during semen processing (Rodriguez-Martinez, 2003). Koonjaenak et al. (2007) found that the overall mean percentage of sperm abnormalities in swamp buffalo bulls was $15 \%$. The overall sperm morphology did not vary statistically across the year. Although the tail abnormalities were affected by season, they were always below what is considered unacceptable for AI bulls. On the other hand, age of bull had a significant $(\mathrm{P}<0.001)$ effect on the incidence of total pathological head shapes, acrosome defects, proximal cytoplasmic droplets and total tail defects. Generally speaking, the sperm defects identified in our study are similar to those that have been described in other livestock species. Different reasons have been proposed as justifications for sperm abnormalities, including genetic factors (Chenoweth, 2005; Mahmoud et al., 2015; Zhang et al., 2015; Dhanoa et al., 2016 and Capra et al., 2017) and temporal impairment of either testicular or epididymal function (Rodriguez-Martinez, 2003). Recently, Bhakat et al. (2015) found different types of chromosomal abnormalities in Murrah buffalo including acrosome integrity as well as head, tail and mid-piece abnormality which averaged 70.10, 2.30, 1.62 and $5.50 \%$, respectively.

Testosterone concentration in peripheral blood demonstrated similar trend to that recorded for semen characteristics, where the estimate recorded for G2 was significantly $(\mathrm{P}<0.05)$ higher than G1 (Table 1). The concentration of testosterone hormone in the present study averaged $4.46 \mathrm{ng} / \mathrm{ml}$, which is lower than that of 5.62 reported by Hafez (2012). The authors found that almost all studied semen traits (semen volume, sperm concentration, total sperm output/ejaculate and live sperm output/ejaculate) varied significantly $(\mathrm{P}<0.05)$ along the experimental period prolonged from April to September due to changes in heat temperature. As the sexual activity of each animal species is highly affected by the surrounding environment, the variation in these parameters was quite obvious on all semen parameters studied by Bhakat et al. (2015). Reasons for good quality seminal ejaculates during winter compared to summer season might be due to the congenial weather conditions which influence the activity and secretions of accessory reproductive glands, since the secretions are dependent on testosterone liberated by interstitial cells during winter which might have favored the spermatogenesis process (Mandal et al., 2005). Semen characteristics and testosterone concentration seem to follow a positive relationship as confirmed by Mandal et al. (2005) and Hafez (2012).

\section{Chromosomal aberrations:}

Table (2) shows different types of chromosomal aberrations detected in blood samples collected from the Egyptian buffalo bulls. Generally speaking, all estimates measured for G2 were significantly $(\mathrm{P}<0.05)$ lower than those recorded for $\mathrm{G} 1$ indicating possible relationship between the observed reproductive performance of the buffalo bulls and the proportion of chromosomal abnormalities they carry.

Within each group of animals, the total percent of numerical aberrations were considerably inferior to their counterpart of structural aberrations. This finding is in agreement with that found by Vijh et al. (1994) in Murrah buffalo, El-Bayomi et al. (2011) in cattle and each of Patel et al. (2012) and Jamir et al. (2015) in Holstein Friesian cattle and Murrah buffalo. Considering numerical aberrations of chromosomes, two types of abnormalities were identified. Polyploidy accounted for $1.2 \%$ of total numerical aberrations in G2 compared to $9.6 \%$ in G1. The second type was aneuploidy that represented $0.4 \%$ and $5 \%$ in $\mathrm{G} 2$ and $\mathrm{G} 1$, respectively. The two studied groups differed significantly $(\mathrm{P}<0.05)$ from each other in the percentages of polyploidy and aneuploidy. El-Bayomi et al. (2011) found that the percent of total numerical aberrations for repeat breeder group in Holstein Friesian cattle was 19.95\% including $9.5 \%$ polyploidy and $10.45 \%$ aneuploidy. The karyotypic profile of normal fertile cows showed that the diploid number of chromosomes is $2 n=60$ chromosomes (29 pairs of acrocentric autosomes and one pair of submetacentric X chromosomes). On the contrary, the water buffaloes (Bubalus bubalis) possess 50 chromosomes, divided into 24 pairs of autosomes and one pair of sex chromosomes (Patel et al., 2012).

In regards to structural chromosomal aberrations, multiple profiles of chromosomal abnormalities were observed. The results obtained in the current study indicated that the total percent of structural aberrations were predominant over the numerical ones and recorded $6.2 \%$ and $45 \%$ in $\mathrm{G} 2$ and $\mathrm{G} 1$, respectively. Figure (1) shows some of the chromosomal aberrations of structural type identified 
in the present study. The structural aberrations included deletion, break, gap, fragmentation, ring, centromeric attenuation and centric fusion. Breeding bulls belonging to $\mathrm{G} 1$ differed significantly $(\mathrm{P}<0.05)$ from those of G2 in all aspects of structural aberrations detected. Except centric fusion, all chromosomal abnormalities of structural origin detected in our study were also identified by ElBayomi et al. (2011) in cattle. Our results are in harmony with that reported by Koonjaenak et al. (2007) in swamp buffalo in Thailand, El-Bayomi et al. (2011) in Holstein Friesian in Egypt and Bhakat et al. (2015) in Murrah buffalo in India. On the other hand, the metaphase plates did not reveal any numerical or structural chromosomal anomaly in Holstein Friesian cattle and Murrah buffalo raised in India (Jamir et al., 2015).

Table 2. Numerical and structural chromosomal aberrations in Egyptian buffalo bulls

\begin{tabular}{lcccccc}
\hline \multirow{2}{*}{ Items } & \multicolumn{3}{c}{ G1 (low semen quality) } & \multicolumn{2}{c}{ G2 (normal semen quality) } \\
\cline { 2 - 7 } & $\begin{array}{c}\text { No. } \\
\text { cells }\end{array}$ & $\begin{array}{c}\text { Aberration } \\
(\%)\end{array}$ & Mean \pm S.E. & $\begin{array}{c}\text { No. } \\
\text { cells }\end{array}$ & $\begin{array}{c}\text { Aberration } \\
(\%)\end{array}$ & Mean \pm S.E. \\
\hline Numerical aberrations & & & & & & \\
Polyploidy & 48 & 9.6 & $0.128 \pm 0.015^{\mathrm{a}}$ & 12 & 1.2 & $0.012 \pm 0.003^{\mathrm{b}}$ \\
Aneuploidy & 25 & 5.0 & $0.040 \pm 0.03^{\mathrm{a}}$ & 4 & 0.4 & $0.001 \pm 0.001^{\mathrm{b}}$ \\
\hline Total & 73 & 14.6 & & 16 & 1.6 & \\
\hline Structural aberrations & & & & & & \\
Deletion & 61 & 12.2 & $0.136 \pm 0.015^{\mathrm{a}}$ & 17 & 1.8 & $0.014 \pm 0.004^{\mathrm{b}}$ \\
Break & 48 & 9.6 & $0.124 \pm 0.015^{\mathrm{a}}$ & 6 & 0.6 & $0.004 \pm 0.002^{\mathrm{b}}$ \\
Gap & 35 & 7.0 & $0.120 \pm 0.015^{\mathrm{a}}$ & 12 & 1.3 & $0.013 \pm 0.004^{\mathrm{b}}$ \\
Fragmentation & 19 & 3.8 & $0.106 \pm 0.014^{\mathrm{a}}$ & 5 & 0.5 & $0.005 \pm 0.002^{\mathrm{b}}$ \\
Ring & 22 & 4.4 & $0.052 \pm 0.009^{\mathrm{a}}$ & 6 & 0.6 & $0.002 \pm 0.001^{\mathrm{b}}$ \\
Centromeric Attenuation & 15 & 3.0 & $0.036 \pm 0.008^{\mathrm{a}}$ & 9 & 0.9 & $0.003 \pm 0.002^{\mathrm{b}}$ \\
Centric Fusion & 25 & 5.0 & $0.132 \pm 0.015^{\mathrm{a}}$ & 5 & 0.5 & $0.007 \pm 0.003^{\mathrm{b}}$ \\
\hline Total & 225 & 45 & & 60 & 6.2 & \\
\hline
\end{tabular}

G1, low semen quality group; G2, normal semen quality group

Values with different super scripts between columns for the same item differ significantly $(\mathrm{P}<0.05)$

A total of 50 cells were examined per each animal

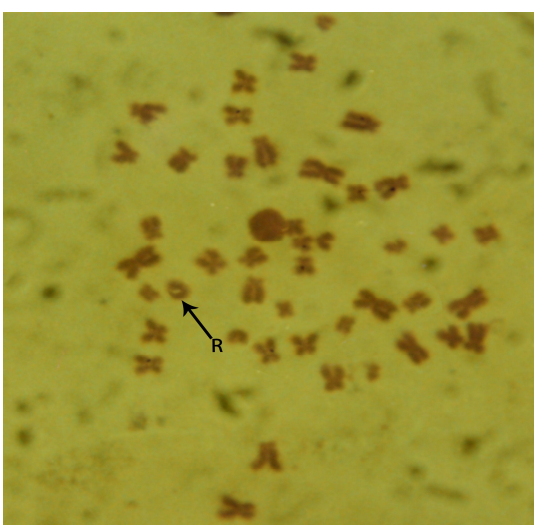

(a)

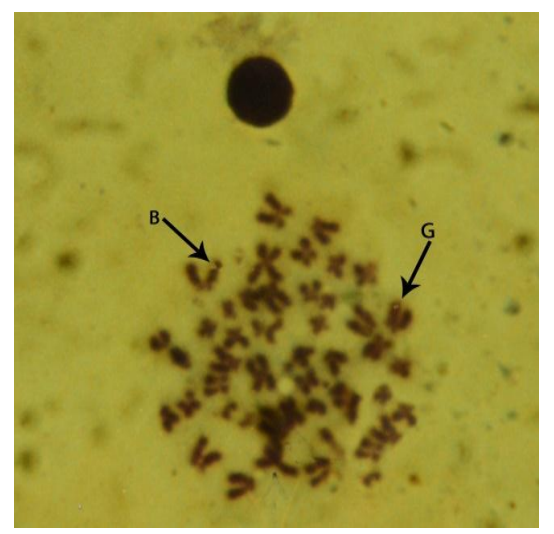

(b)

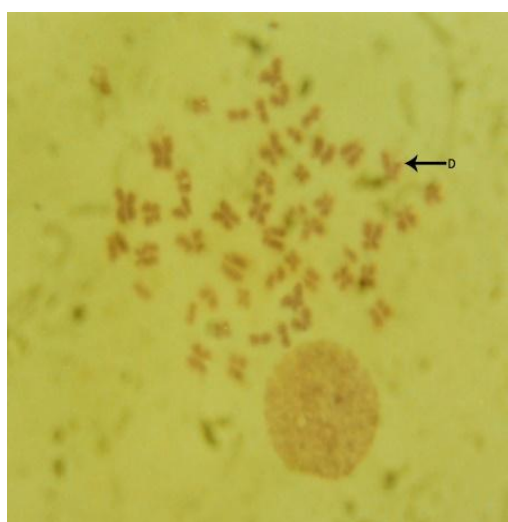

(c)

Figure 1. Metaphase spreads of buffalo bulls: (a) ring, (b) break \& gap and (c) deletion

The Y- and X-chromosome in mammalian species have evolved from autosomes and are involved in sex determination and reproductive traits. The Y-chromosome plays major role in male fertility and is suitable to study the evolution, speciation and male infertility and/or subfertility due to its unique features such as long non-recombining region, abundance of repetitive sequences and holandric inheritance pattern (Dhanoa et al., 2016).

In the present study, buffalo bulls were initially classified into two groups according to the semen quality. Obviously, animals of G2 had better semen characteristics than those of G1, and also G2 bulls demonstrated to have lower cases of chromosomal aberrations in comparison to G1 bulls. Consequently, it is expected that the data available on semen quality and chromosomal abnormalities would depict the future reproductive performance of those young buffalo bulls. Almost all the sex chromosome abnormalities shown in river buffalo were correlated to sterility (Patel et al., 2012 and Yimer and Rosnina, 2014). The results obtained in the current study encourage the incorporation of the clinical cytogenetics for investigation of chromosomal anomalies in young buffalo bulls prior to being used in artificial insemination and breeding programs. 
Unlike cattle, systematic cytogenetic screening programmes for chromosomal aberrations in both river and swamp buffaloes are not common and most of the reports are based on individuals suffering from reproductive problems. Autosomal chromosome abnormalities have been less reported in river buffalo compared with cattle; however, the most common chromosome aberration reported in river buffalo is sex chromosome abnormality (Yimer and Rosnina, 2014).

\section{Genetic correlation:}

Correlation coefficients between semen characteristics and chromosomal abnormalities are given in Table 3. All estimates of genetic correlation were significant $(\mathrm{P}<0.05)$, except that related to centromeric attenuation. Motility, live sperm, hypoosmotic swollen (\%) and testosterone level had negative correlation coefficients with those traits associated with numerical and structural chromosomal aberrations. These findings indicate that selection that favors one trait can cause a decrease in other traits due to their negative correlation. The estimates obtained in the present study are generally lower than those reported in the Egyptian buffalo by Khattab et al. (2015) and Mahmoud et al. (2015).

Table 3. Coefficients of genetic correlation between semen-related traits and chromosomal aberrations in Egyptian buffalo bulls

\begin{tabular}{lccccccccc}
\hline & PO & AN & D & B & G & F & R & CA & CF \\
\hline MO & -0.391 & -0.236 & -0.322 & -0.187 & -0.232 & -0.250 & -0.238 & -0.092 & -0.250 \\
LI & -0.350 & -0.230 & -0.317 & -0.171 & -0.181 & -0.279 & -0.224 & -0.103 & -0.238 \\
AB & 0.341 & 0.242 & 0.334 & 0.221 & 0.176 & 0.251 & 0.251 & 0.078 & 0.261 \\
HOS & -0.372 & -0.261 & -0.322 & -0.195 & -0.215 & -0.250 & -0.220 & -0.126 & -0.235 \\
TEST & -0.294 & -0.263 & -0.262 & -0.192 & -0.272 & -0.254 & -0.183 & -0.125 & -0.175 \\
\hline
\end{tabular}

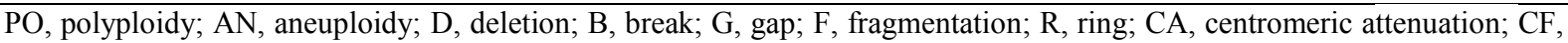
centric fusion; MO, motility; LI, live sperm; $\mathrm{AB}$, abnormality; HOS, hypo-osmotic swollen (\%); TEST, testosterone hormone

In this respect, Ostermeier et al. (2001) studied the relationship between sperm nuclear shape and fertility using two groups of bulls differing in fertility based on lifetime non return rates. The results indicated that mean nuclear shape of high-fertility sperm was more elongated and tapered than those of low-fertility sperm. Also, a comparison of sperm chromatin structure analysis and harmonic amplitudes found that various measures of sperm nuclear shape were correlated significantly $(\mathrm{P}<0.05)$ to chromatin stability. The authors suggested that sperm nuclear shape is a better predictor of bull fertility because some of the differences observed in sperm nuclear shape between the high- and lowfertility bulls may be due to different levels of chromatin stability.

In contrast, sperm abnormalities showed positive estimates of genetic correlation with chromosomal aberrations, ranging from 0.078 to 0.341 for centromeric attenuation and polyploidy, respectively. Correlation occurs when one trait is selected for, and another trait is changed simultaneously. The positive coefficients between sperm abnormalities and all chromosomal abnormalities would result in a correlated response when selecting for one trait only. Therefore, early selection for normal karyotype solely may ensure good semen characteristics at maturity. Our results are in consistency with those of earlier studies, in which chromosome profile was correlated with sperm morphometric values (Ostermeier et al., 2001; Ahmad et al., 2004; Katragadda et al., 2013 and Mahmoud et al., 2015). In swamp buffalo, Koonjaenak et al. (2007) reported very low correlations between sperm chromatin integrity and sperm head morphology, where the most significant one was between DNA fragmentation index and loose abnormal heads.

Many studies indicated that different forms of chromosomal aberrations influenced fertility of buffalo and cattle (Chauhan et al., 2009; Patel et al., 2012; Katragadda et al., 2013; Yimer and Rosnina, 2014 and Dhanoa et al., 2016). As reported by Mahmoud et al. (2015), motility and live sperm (\%) correlated significantly $(\mathrm{r}=0.482, \mathrm{P}<0.05)$. Also, they observed an opposite trend concerning motility and sperm abnormalities $(\mathrm{r}=-0.64, \mathrm{P}<0.05)$ as well as motility and DNA damage $(\mathrm{r}=-0.68, \mathrm{P}<0.05)$, whereas a significant positive correlation $(\mathrm{r}=0.59, \mathrm{P}$ $<0.05$ ) was reported between sperm abnormalities and DNA fragmentation. The authors concluded that motility may be a physiological marker of choice for semen quality because significant correlations were obtained between motility and other sperm parameters including sperm abnormalities and DNA damage. Moreover, on the light of the significantly positive correlation $(\mathrm{r}=0.59, \mathrm{P}<0.05)$ found between sperm abnormalities and DNA fragmentation, the authors concluded that evaluating DNA damage can offer a simple description of DNA integrity and chromosomal aberrations which can predict the quality of sperms used in artificial insemination. Similar findings were reported by Ramos and Wetzels (2001) and Capra et al. (2017). According to Irvine et al. (2000), sperm motility is considered as an indicator of semen quality and conception rate when artificial insemination used due to motility reflects chromatin integrity and DNA fragmentation. The results indicated that sperm 
samples with low motility tended to carry higher loads of DNA damage.

In a study to assess reasons of poor reproductive performance in buffalo and cattle bulls, Jamir et al. (2015) indicated that metaphase plates did not show any structural or numerical chromosomal abnormalities in both species. High and low motility groups within both buffalo and cattle revealed significant $(\mathrm{P}<0.05)$ differences in HOST. However, the two species did not differ significantly from each other. In spite of not detecting any chromosomal abnormalities, the authors recommend testing future breeding bulls for cytogenetic profile and sperm membrane integrity.

Interestingly, Schmutz et al. (1996) found that about $10.7 \%$ of 55 aborted fetuses and stillborn calves belonging to 12 different dairy and beef cattle breeds had an abnormal chromosome complement. In about $5.5 \%$ of such cases, the abnormalities were probably the cause of death that usually happened during the last month of gestation period. This finding supports the adoption of chromosomal analysis as a cost effective tool to diagnose expected malformed fetuses and newborns. Because of seasonal suppression of fertility repeat breeding in buffaloes should be limited to the breeding season. Spring and winter calving, first parity, periparturient disease and lactation are significant risk factors for repeat breeding in buffaloes (Saraswat and Purohit, 2016). Patel et al. (2012) studied the relationship between chromosomal abnormalities and fertility problems in cattle and water buffalo breeding bulls. They found light incidences of chromosomal abnormalities in a few samples, including polyploidy, premature centromeric division, chromosomal fragmentation, aneuploid cells, fragile site, chromatid gaps or breaks in both species. As the detected aberrations were not consistent features in a particular animal; therefore, those aberrations could not have great effects on fertility. However, the authors suggested applying a regular screening to score such chromosomal aberrations which might cause a reduction in animal fertility. More recently, Mughal et al. (2017) found that the spermatozoa motility, acrosomal integrity and DNA integrity were significantly $(\mathrm{P}<0.05)$ influenced by varied osmotic pressures.

Heritability of semen quality traits was estimated by Khattab et al. (2015). They found that heritability estimates for volume of semen, livability of sperm and motility of sperm were $0.30,0.38$ and 0.35 , respectively. Moreover, estimates of genetic correlations between each pair of these traits were positive and highly significant $(\mathrm{P}<0.05)$. The expected genetic change per generation ranged from 0.45 to $1.50 \mathrm{ml}$ for volume of semen, from 6.19 to $10.50 \%$ for livability and from 6.21 to 7.22 for motility of sperm. The authors suggested incorporating volume of semen in a selection index that involves motility and/or livability of sperm.

The relationship between chromosomal aberrations and reduced fertility in livestock has been widely studied for decades (Koonjaenak et al., 2007; Saraswat and Purohit, 2016 and Dhanoa et al., 2016). According to Raudsepp and Chowdhary (2016), chromosomal abnormalities directly affect meiosis, gametogenesis, and the viability of zygotes and embryos. In some instances, balanced structural rearrangements can be transmitted, causing fertility problems in subsequent generations.

Traditionally, the chromosomal abnormalities were reported basically by conventional methods (banding of chromosomes) by which it was difficult to detect specific causes of aberrations and to predict accurately bull fertility. The new molecular technologies like probe-based fluorescence in situ hybridization (FISH), sequencing and microarray may be applied to identify minor chromosomal abnormalities so that they can be correlated with infertility problems. In this respect, a method based on deep sequencing of sperm microRNA (miRNA) and Piwi-interacting RNA (piRNA) from individual bulls was recently developed by Capra et al. (2017). The specific miRNA expression was differentially represented in low versus high motile sperm, indicating an alteration of cell functions and increased germ cell apoptosis in the low motile fraction (Raudsepp and Chowdhary, 2016).

Currently, traditional cytogenetics is interfaced with advanced genomics approaches, such as array technologies and next-generation sequencing, to enrich our knowledge about animal genomes and consequently improve our understanding of connections between gross structural or molecular chromosome variations and reproductive disorders in domestic animals.

Generally speaking, significant differences in chromosomal aberrations and semen quality were found in the present study between low and normal semen quality categories. It can be concluded the possible association of semen characteristics with chromosomal aberrations, and subsequently the ability to incorporate it in early selection schemes for fertility in the Egyptian buffalo bulls. The breeding buffalo bulls kept for semen production have to be screened for cytogenetic profile and the sperm membrane integrity measured as HOST.

\section{ACKNOWLEDGEMENT}

Financial support provided by Animal Production Research Institute, Dokki, Giza, Egypt is acknowledged.

\section{REFERENCES}

Ahmad I., K. Javed and A. Sattar, 2004. Screening of breeding bulls of different breeds through karyotyping. Pakistan Vet. J., 24 (4): 190-192.

Amann R. P. and R. H. Hammerstedt, 1980. Validation of a system for computerized measurements of spermatozoa velocity and percentage of motile sperm. Biol. Repro., 23 (3): 647-656. 
Azam M., M. Anzar and M. Arslan, 1998. Assessment of post-thaw semen quality of buffalo and sahiwal bulls using new semen assays. Pakistan Vet. J., 18 (2): 74-80.

Barkawi A. H., G. Ashour, Y. M. Hafez, S. A. Ibrahim, A. K. El-Asheeri and Sh. M. Desoky, 2006. Plasma membrane integrity and acrosomal damage of Egyptian buffalo (Bubalus bubalis) spermatozoa in relation to period of the year, freezing and type of extender. Buffalo J., 2: 143153.

Bhakat M., T. K. Mohanty, A. K. Gupta, S. Prasad, A. K. Chakravarty and H. M. Khan, 2015. Effect of season on semen quality parameters in Murrah buffalo bulls. Buffalo Bulletin, 34 (1): 100-112.

Blom E., 1983. The spermiogram of the bull. Nordisk Vet. Med., 35: 105-111.

Capra E., F. Turri, B. Lazzari, P. Cremonesi, T. M. Gliozzi, I. Fojadelli, A. Stella and F. Pizzi, 2017. Small RNA sequencing of cryopreserved semen from single bull revealed altered miRNAs and piRNAs expression between high- and low-motile sperm populations. BMC Genomics, 18: 14.

Chauhan J. B., R. K. Patel, K. M. Singh and K. J. Soni, 2009. Impact of a novel cytogenetic finding (usual XX translocation) on fertility of buffalo bulls (Bubalus bubalis). Buffalo Bulletin, 28 (3): 151-153.

Chenoweth P. J., 2005. Genetic sperm defects. Theriogenology, 64: 457-468.

Dhanoa J. K., C. S. Mukhopadhyay and J. S. Arora, 2016. Y-chromosomal genes affecting male fertility: A review. Veterinary World, 9 (7): 783 791.

El-Bayomi Kh. M., I. E. El-Araby and A. W. Zaglool, 2011. Cytogenetic analysis related to some infertility problems in cattle. Global Veterinaria, 7 (4): 323-329.

FAOSTAT "Online statistical service of the Food and Agriculture Organization of the United Nations". 2016. (Available online with updates at http://faostat3.fao.org).

Galal S., 2007. Farm animal genetic resources in Egypt: factsheet. Egyptian J. Anim. Prod., 44: 123.

Hackett A. J. and J. W. Macpherson, 1965. Some staining procedures for spermatozoa. A review. Can. Vet. J., 6 (3): 55-62.

Hafez Y. M., 2012. Physicochemical characteristics of semen from buffalo bulls (Bubalus bubalis) as affected by treatment of two different doses of recombinant bovine somatotropin (rbST). Egy. J. Anim. Prod., 49 (3): 241-248.

Halnan C. R. E., 1977. An improved technique for the preparation of chromosomes from cattle whole blood. Research Vet. Sci., 22 (1): 40-43.

Iannuzzi L. and D. Di Berardino, 2008. Tools of the trade: diagnostics and research in domestic animal cytogenetics. Journal of Applied Genetics, 49 (4): 357-366.

Irvine D. S., J. P. Twigg, E. L. Gordon, N. Fulton, P. A. Milne and R. J. Aitken, 2000. DNA integrity in human spermatozoa: relationships with semen quality. J. Androl., 21 (1): 33-44.

Jamir C. J., C. S. Mukhopadhyay, J. S. Arora, G. S. Brah, R. Cheema and M. Kaur, 2015. Screening of dairy breeding bulls for chromosomal profile and its andrological attributes. Indian J. Anim. Res., 49 (1): 8-13.

Katragadda S., R. K. Patel and R. Kotikalapudi, 2013. Blood cell chimerism (XX/XY) in Murrah buffalo bulls. Bang. J. Anim. Sci., 42 (1): 20-22.

Khattab A. S., H. S. Zeweil, M. H. Ahmed, S. M. Zahran, Y. M. El-Gindy, A. Z. Mohamed and K. A. Mourd, 2015. A comparison of different selection indexes for some semen traits on Egyptian buffalo bulls. Middle East Journal of Applied Sciences, 5 (2): 368-373.

Koonjaenak S., V. Chanatinart, S. Aiumlamai, T. Pinyopumimintr and H. Rodriguez-Martinez, 2007. Seasonal variation in semen quality of swamp buffalo bulls (Bubalus bubalis) in Thailand. Asian J. Androl., 9 (1): 92-101.

Mahmoud K. Gh. M, A. A. E. El-Sokary, A. E. Abdel-Ghaffar, M. E. A. Abou El-Roos and Y. F. Ahmed, 2015. Analysis of chromatin integrity and DNA damage of buffalo spermatozoa. Iranian Journal of Veterinary Research, 16 (2): 161-166.

Mandal D. K., S. K. Tyagi and A. K. Mathur, 2005. Semen production performance of Sahiwal bulls. Indian J. Anim. Sci., 75 (1): 17-19.

Mughal D. H., A. Ijaz, M. S. Yousaf, F. Wadood, U. Farooq, S. A. Mahmood and A. Riaz, 2017. Effect of osmotic pressure on spermatozoa characteristics of cryopreserved buffalo bull (Bubalus bubalis) semen. J. Appl. Anim. Res. (http://dx.doi.org/10.1080/09712119.2017.129597 1).

Ostermeier G. C., G. A. Sargeant, B. S. Yandell, D. P. Evenson and J. J. Parrish, 2001. Relationship of bull fertility to sperm nuclear shape. J. Androl., 22 (4): 595-603.

Patel D. J., A. J. Patel, R. K. Patel and P. R. Parekh, 2012. Chromosomal analysis of breeding bulls using lymphocyte culture. The Bangladesh Veterinarian, 29 (1): 17-21.

Ramos L. and A. M. Wetzels, 2001. Low rates of DNA fragmentation in selected motile human spermatozoa assessed by the TUNEL assay. Hum. Reprod., 16 (8): 1703-1707.

Rasul Z., N. Ahmad and M. Anzar, 2001. Changes in motion characteristics, plasma membrane integrity, and acrosome morphology during cryopreservation of buffalo spermatozoa. J. Androl., 22 (2): 278-83.

Raudsepp T. and B. Chowdhary, 2016. Chromosome aberrations and fertility disorders in domestic animals. Annual Review of Animal Biosciences, 4: $15-43$.

Rodriguez-Martinez H., 2003. Laboratory semen assessment and prediction of fertility: still utopia? Reprod. Domest. Anim., 38 (4): 312-318.

Saraswat C. S. and G. N. Purohit, 2016. Repeat breeding: Incidence, risk factors and diagnosis in 
buffaloes. Asian Pacific Journal of Reproduction, 5 (2): 87-95.

Schmutz S. M., J. S. Moker, E. G. Clark and J. P. Orr, 1996. Chromosomal aneuploidy associated with spontaneous abortions and neonatal losses in cattle. J. Vet. Diagn. Invest., 8 (1): 91-95.

Smith O. W. and H. D. Hafs, 1973. Competitive protein binding and radioimmunoassay for testosterone in bulls and rabbits: Blood serum testosterone after injection of $\mathrm{LH}$ or prolactin in rabbits. Proceedings of the Society for Experimental Biology and Medicine, 142 (3): 804-810.

SPSS 14, 2006. Statistical Package for Social Science, SPSS for windows release 14.0.0, 12
June, 2006, all rights reserved, copyright ${ }^{\circledR}$ SPSS Inc.

Vijh R. K., M. S. Tanita and R. Sahai, 1994. Translocation in Murrah buffalo. Indian J. Anim. Sci., 64: 534-538.

Yimer N. and Y. Rosnina, 2014. Chromosomal anomalies and infertility in farm animals: A review. Pertanika J. Trop. Agri. Sci., 37 (1): 1-18.

Zhang M., H. T. Fan, Q. S. Zhang, X. Y. Wang, X. Yang, W. J. Tian and R. W. Li, 2015. Genetic screening and evaluation for chromosomal abnormalities of infertile males in Jilin Province, China. Genetics and Molecular Research, 14 (4): 16178-16184.

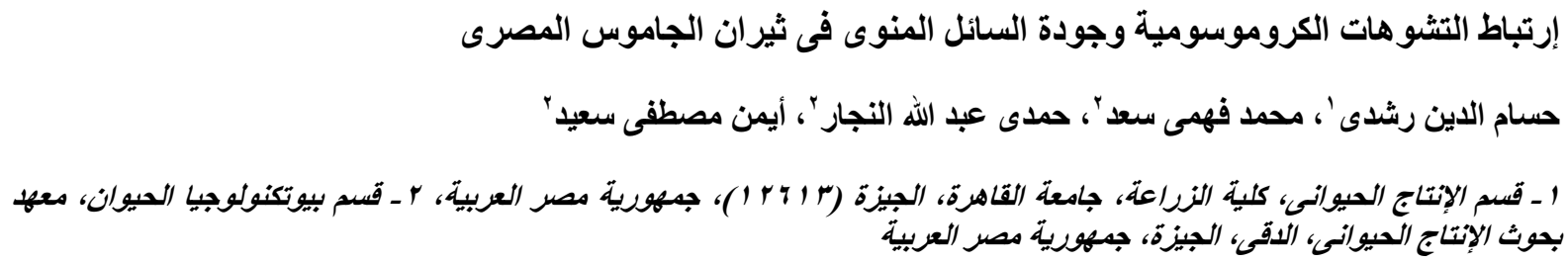

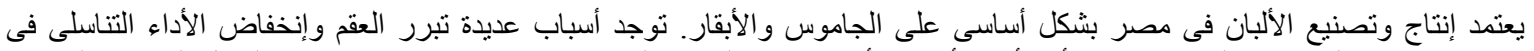

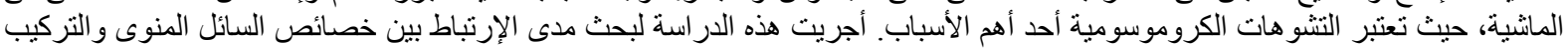

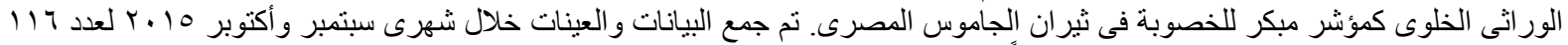

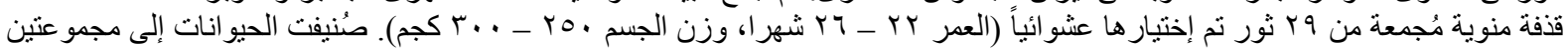

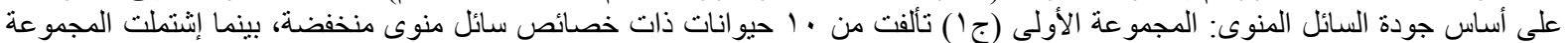

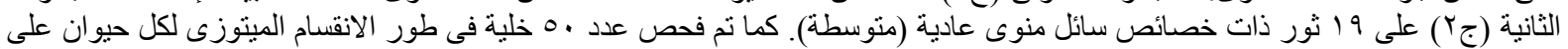

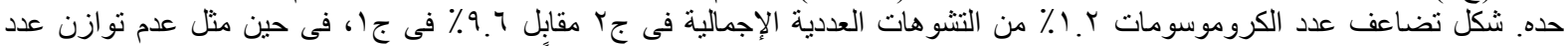

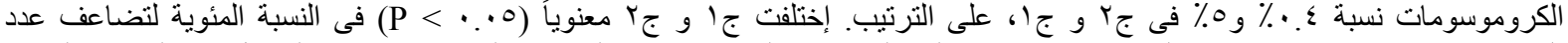

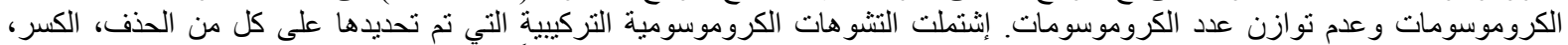

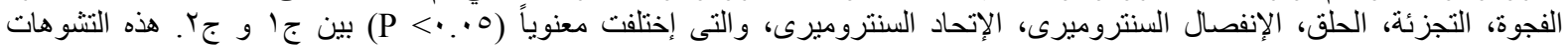

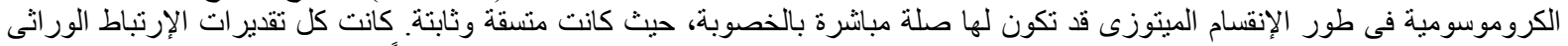

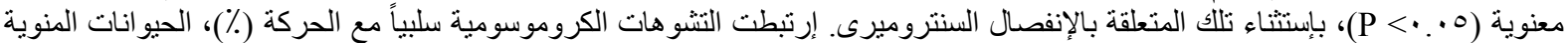

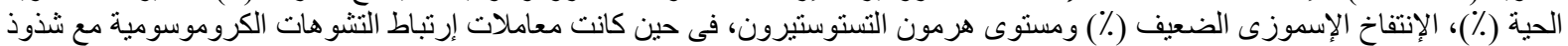

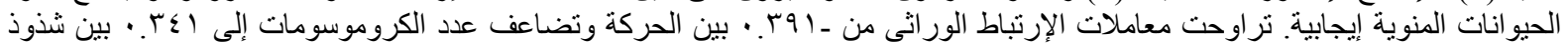

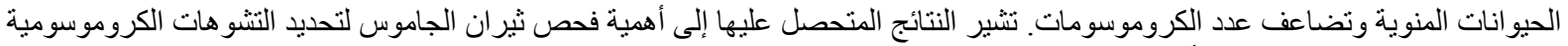
قبل إستخدامها فى التلقيح الطبيعى أو الإصطناعى. 